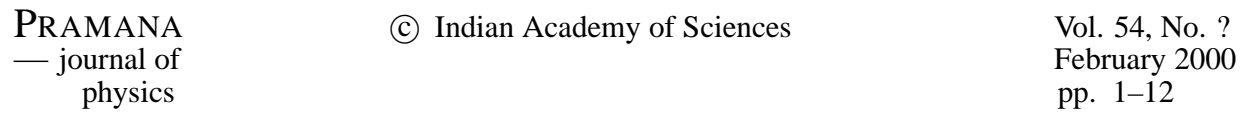

\title{
Novel interference effects and a new Quantum phase in mesoscopic systems
}

\author{
P. Singha Deo* \\ S. N. Bose National Center for Basic Sciences, JD Block, Sector III, Salt Lake City, Calcutta, India
}

\author{
A. M. Jayannavar ${ }^{+}$ \\ Institute of Physics, Sachivalaya Marg, Bhubaneswar - 751 005, India
}

\begin{abstract}
.
Mesoscopic systems have provided an opportunity to study quantum effects beyond the atomic realm. In these systems quantum coherence prevails over the entire sample. We discuss several novel effects related to persistent currents in open systems which do not have analogues in closed systems. Some phenomena arising simultaneously due to two non-classical effects namely, AharonovBohm effect and quantum tunneling are presented. Simple analysis of sharp phase jumps observed in double-slit Aharonov-Bohm experiments is given. Some consequences of parity violation are elaborated. Finally, we briefly describe the dephasing of Aharonov-Bohm oscillations in Aharonov-Bohm ring geometry due to spin-flip scattering in one of the arms. Several experimental manifestations of these phenomena and their applications are given.
\end{abstract}

Keywords. mesoscopic systems,coherence,Aharonov-Bohm effect,persistent currents,parity.

PACS Nos 2.0

\section{Introduction}

Mesoscopic physics deals with samples that are intermediate in size between the atomic scale and the macroscopic scale determined by the transport phase coherence length of electrons or quasiparticles. Studies in these systems have revealed a new range of unexpected quantum phenomena, often counter-intuitive. Interpretation of these phenomena requires full recognition of the wave nature of quasiparticles and keeping track of their phase coherence over the entire sample including the measurement leads and probes (quantum measurement process). Clearly, when the transport dimension reaches the chargecarrier inelastic scattering length (coherence length) and charge confinement dimension approaches Fermi wavelength, then the physics of these systems, based on the motion of particles and ensemble averaging is expected to be invalid. The notion of the usual ensemble averaged transport coefficient such as the resistivity/conductivity, that is local and material specific, has to be replaced by that of resistance/conductance, that is global and operationally specific to the sample as well as the nature of probe for measurements. 
The guiding theme of mesoscopic physics is the quantum interference over the entire sample or treating the whole mesoscopic sample as a single quantum scatterer. These systems exhibit properties where interference of electronic waves, quantization of energy levels (quantum size effects), discreteness of charge and their number being even or odd (parity effects) play a major role. Thus we have an opportunity for exploring truly quantum effects beyond the atomic realm. This subject enjoys the unique position of being able to deal with and provide answers on fundamental questions in physics while being relevant to applications in the area of quantum electronics, computation and communication which are rapidly emerging fields in their own respect. Basic questions about how the quantum rules operate and go over to classical ones at the macroscopic level as one tunes the temperature or sample size are being answered, i.e., mesoscopic systems present the possibility of studying in a controlled way the process of decoherence (or dephasing) and the transition from quantum to classical behavior.

Some of the experimentally observed phenomena in these systems include breakdown of classical Ohm's law [1], the normal state Aharonov-Bohm (AB) oscillations in resistance [2], normal electron persistent currents [3], non-local current and voltage relations (manifestation of quantum non-locality) [4], conductance quantization of point contacts [1], normal and anomalous quantum Hall effect, negative four probe resistances, quantum shot noise, single electron transistor (Coulomb blockade), proximity effect in mesoscopic superconductors, spin coherence effects (spintronics), entangled states in quantum dots etc. to name but a few. Interestingly, many of these phenomena can be observed through the use of straight forward experimental probes, namely the dc electrical two probe and four probe conductance in the presence or absence of magnetic field.

The propagation of electron has many interesting similarities with other wave propagation such as electromagnetic wave or sound wave propagation etc. and thus mesoscopic phenomenon can be understood in terms of wave guide theory. In this work we will discuss various mesoscopic phenomena that we studied using the mode matching technique for wave guides. Such a study can also be performed using Greens function techniques or using the tight binding Hamiltonian, but for non-interacting systems the mode matching technique allows more explicit and exact calculations. Quantities like conductance, local and global currents and eigenenergies can be calculated from first principles. Most of the phenomena discussed here are new and were pointed out by us. Some of them have experimental consequences as well as applications in quantum devices.

In the following sections we discuss several novel effects related to persistent currents and transport in open systems (connected to external reservoirs), which have no analogue in closed or isolated systems. Some of the effects which we discuss arise simultaneously due to two non-classical effects namely AB-effect and quantum tunneling. We also discuss the observed additional new phase of electron wavefunction in the AB-ring geometry, breakdown of parity effects and their consequences. Finally we briefly discuss our ongoing work on dephasing of $\mathrm{AB}$-oscillations in the presence of a magnetic impurity (leading to exchange spin flip scattering) in one of the arms of the AB-ring. This has resemblance with which-way interferometer or which-path detector models developed to study dephasing due to quantum measurement process. 


\section{Directional dependence of persistent currents and the quantum current magnifica- tion effect}

Persistent currents in closed loops are equilibrium quantum mechanical currents that were first predicted [5] and subsequently detected in normal metals [3] and semiconductor [6] rings. Such currents were theoretically known for a very long time [7]. The application of magnetic field ( $\mathrm{AB}$ flux) destroys the time reversal symmetry and as a consequence persistent currents flow in the loop and are periodic in magnetic flux, with a period $\phi_{0}$, $\phi_{0}=h c / e$ being the elementary flux quanta. For a perfect ring of circumference $L$ the magnetic flux $\phi$ enclosed by the loop modifies the periodic boundary condition into $\psi(x+L)=\psi(x) e^{i 2 \pi \phi / \phi_{0}}$. This tuning of boundary condition changes the energy levels in a periodic manner. Persistent current, being the flux derivative of the total free energy, is also periodic in flux. Thus persistent current can be attributed to changing boundary conditions due to magnetic flux. At zero temperature for spinless electrons persistent current is diamagnetic or paramagnetic depending on the number of electrons in the loop being odd or even respectively (parity effect). It was subsequently shown $[8,6]$ that persistent currents can also occur in open systems i.e., when the system can exchange electrons with electron reservoirs. In such open systems, apart from persistent currents one can also have transport currents. While transport currents are non-equilibrium currents and require a potential difference (voltage difference, temperature gradients or chemical potential difference), persistent currents are local internal currents that do not explicitly depend on the potential difference. We show that one can distinguish between these two currents using the two geometries shown in Fig. 1 (a) and (b). These two currents, although exist simultaneously, are fundamentally different. These geometries help to probe their different properties.

The quantum mechanical scattering wave functions can be explicitly written down (depending on the direction of current) in different regions and can be matched at the junctions using Griffith's boundary conditions [9]. We will be considering for simplicity one dimensional free electron networks. Appropriate wave functions in the different regions are thus linear combinations of simple plane wave solutions. The effect of magnetic field can be incorporated in the boundary conditions. From the wave functions one can also calculate the currents in different regions. In case of Fig. 1 (a) one can only have persistent currents in the loop while the transport currents flow in the wires between reservoirs 1 and 2 at chemical potentials $\mu_{1}$ and $\mu_{2}$. In case of Fig. 1 (b) there will be persistent currents as well as transport currents in the loop. The two can be separated because persistent current is an odd function of the flux $\phi$ while the transport current (being proportional to transmission coefficient) is an even function of the flux $\phi$. Apart from being an even function of flux, the transport current also has a flux independent part but the persistent current by definition has no flux independent part. Once the two currents are separated using these basic properties, we find that the magnitude of the transport current is independent of the direction of the current flow between the reservoirs (conductance of the sample is the same whether $\mu_{1}>\mu_{2}$ or vice versa). But the magnitude of the persistent current in the loop depends on the direction of the direct inter-reservoir current. The defect at the position X plays a very important role in realizing this directional dependence of persistent currents. The defect breaks the spatial symmetry in the problem. Depending on the direction of current flow (for different scattering problem) we will have a different complex amplitude of wave function at the junction between loop and the wire. This amounts to changing the 
boundary condition. As discussed above the persistent currents being sensitive to boundary conditions will have different magnitudes. For another extreme example when the defect strength becomes infinite then reservoir 2 is cut off and does not contribute to the persistent current in the loop. Hence the condition whether $\mu_{1}>\mu_{2}$ or not will definitely make a difference. The directional independence of conductance is a fundamental property of two probe Landauer conductance and most quantities like noise and fluctuations are always independent of direction of the current flow. In this respect the properties of persistent currents in open systems is very unique.

Next we consider a situation when the impurity at site X in Fig. 1 (b) and the AharonovBohm flux are not essential but the lengths of the two arms of the loop $L_{1}$ and $L_{2}$ are different. Asymmetry in the two arms of the ring is a must to obtain this quantum effect. When the two arms are identical in all respects then half the transport current flows through the upper arm and the rest half through the lower arm i.e., $I / 2=I_{1}=I_{2}>0$. Here $I$ is the magnitude of the total inter-reservoir current or the transport current and $I_{1}$ and $I_{2}$ are the magnitudes of currents in the two arms of the ring. In the case of classical wires when the arms are not identical then we have the condition $I_{1} / I_{2}=R_{2} / R_{1}$ and $I=I_{1}+I_{2}$ (current conservation or Kirchoff's law) which automatically implies $0<I_{1}<I$ and $0<I_{2}<I$. Here $R_{1}$ and $R_{2}$ are the resistances of the two arms of the ring. Both $I_{1}$ and $I_{2}$ are positive and flow in the same direction of applied fields. But for quantum wires, a simple scattering solution of the Schrodinger equation shows that the above conditions break down. At certain Fermi energies and values of loop parameter one can realize such situations as $I_{1}>I$ which automatically requires $I_{2}<0$, because the current conservation warrants $I=I_{1}+I_{2}$. Thus the current $I_{2}$ flows against the potential drop. Such a phenomenon occurs in the vicinity of resonances in the ring and is quantum mechanical in origin. In classical parallel resonant LCR circuits (capacitance C connected in parallel with a combination of inductance $\mathrm{L}$ and resistance $\mathrm{R}$ ) driven by external electromotive force, circulating currents arise at resonant frequency. This effect is sometimes referred to as current magnification. Hence we call the phenomenon discussed above as "quantum current magnification effect" which gives a circulating current that we define as follows. If both $I_{1}$ and $I_{2}$ are positive or flow in the same direction of the potential drop then the circulating current is zero. If one of them, say $I_{1}$ is negative then circulating current is $I_{1}$ in both the arms. Of course in the lower arm it is in the direction of potential drop but not in the upper arm. $I-I_{1}$ is the transport current flowing in the lower arm in addition to the circulating current $I_{1}$. The parameter regimes where this circulating current exists can be found in Ref. [10]. Experimentally, it is possible to observe this current magnification effect as a large magnetic response of the ring by properly tuning either the Fermi energy or other material parameters. This is due to the fact that magnetic moment of a loop is proportional to the total integration of the current over the entire loop. It should be noted that we are obtaining magnetic response in the absence of applied external magnetic field, however, in the presence of a transport current. This is a non-equilibrium phenomenon.

Now it can also be shown that unlike the conventional persistent currents, the quantum current magnification effect can be enhanced by impurity scattering in certain range of parameter values. To understand that such an effect is possible consider, for example, a case when the two arms of the ring are identical in all respect. In that case by symmetry we have $I_{1}=I_{2}=I / 2>0$ and hence there is no quantum current magnification effect. Now if we introduce an impurity potential (say a delta function potential) in the upper arm of the ring then the symmetry is destroyed and one can thus in principle obtain a current 
magnification effect. This simple example shows that one can have enhancement of current magnification effect due to the impurity scattering. Although this is not a general feature but an extremely parameter dependent one, nevertheless for any configuration of the two arms of the ring one can always find parameter regimes where the circulating current can be enhanced by impurity scattering. In the parameter regimes other than these the circulating current will decrease due to scattering. A detailed analysis of this can be found in Ref. [11]. We have also shown that there is no upper bound for the current magnification. This effect has been extended to thermal currents [12] and to the spin currents in the presence of Aharonov-Casher flux [13].

\section{Persistent currents and transport due to evanescent modes in the presence of AB- flux}

Let us imagine a geometry where a metallic loop is coupled to a single electron reservoir via an ideal lead (Fig. 2). In the ideal lead the potential is assumed to be zero, i.e., $\mathrm{V}=0$. In the metallic loop the potential throughout the circumference is $\mathrm{V}$ and is positive. When injected electrons have their energies less than $\mathrm{V}$, these electrons can tunnel into the loop quantum mechanically and propagate inside the loop as evanescent modes and give rise to a persistent current in the presence of a magnetic field. Such currents arise simultaneously due to two non-classical effects, namely, quantum tunneling and Aharonov-Bohm effect. Currents due to such evanescent modes are to be found by analytical continuation and we have obtained an analytical expression for these persistent currents [14]. In the limit $\mathrm{QL}>>1$, persistent currents in a small energy interval around $\mathrm{E}$ are given by $d j=f(k, Q) e^{-Q L} \sin \left(\phi / \phi_{0}\right)$, where $\mathrm{f}(\mathrm{k}, \mathrm{Q})$ is a simple function of $\mathrm{k}$ and $\mathrm{Q}$. Here $\mathrm{k}$ is the

wave vector for incident electrons, i.e., $\mathrm{k}=\sqrt{2 m E / \hbar^{2}}, \mathrm{Q}=\sqrt{2 m(V-E) / \hbar^{2}}$ and $L$ is the circumference of the loop. As expected the persistent currents are periodic in magnetic flux with the period $\phi_{0}$. Owing to the decaying nature of evanescent modes, the factor arising due to the sensitivity of the wavefunctions to the boundary conditions appears as $e^{-Q L}$. Higher harmonics in magnetic flux (say nth harmonic) also contribute to the persistent currents with a multiplication factor $\sin \left(n \phi / \phi_{0}\right)$. However, these harmonics are weighted by $e^{-n Q L}$ because for these harmonics to appear the electron has to traverse the loop $\mathrm{n}$ times. So, these harmonics can be neglected in the limit QL $>>1$. Unlike the behavior of persistent currents above the barrier regime the currents due to evanescent modes do not oscillate as a function of the Fermi energy as long as $\mathrm{E}<\mathrm{V}$. The total persistent current is given by sum of contributions from the electrons up to Fermi energy. Even though the current due to individual evanescent modes is small the total sum can have an observable amplitude. Especially in a real physical situation one can have a ring with extremely narrow width connected to the reservoir via an ideal wire with a much larger width. In this situation the zero point quantum potential due to the transverse confinement in the ring is much higher than the zero point energy of the ideal wire. Electrons can occupy several subbands in the connecting wire but still they have energies less than the zero point energy of the ring. All these electrons in several subband modes will propagate as evanescent modes in the ring, and in this situation a higher contribution to the total persistent current may arise.

Having shown that persistent currents due to evanescent modes in open systems is always diamagnetic, it would be interesting to see if the same is true in closed systems. One can excite evanescent modes throughout the circumference of the ring in the follow- 
ing closed system. Consider the case of one dimensional loop with higher potential $V$ connected to a stub of length $L$ for which the reference potential is taken to be zero (see Fig. 3). For a sufficiently large value of $V$ and long stub there can be many modes that are propagating in the stub but are classically forbidden in the ring. However, quantum mechanical tunneling leads to evanescent modes in the ring. It has been shown that these discrete evanescent modes always carry a diamagnetic persistent current [15].

As mentioned in the introduction, similarity with the guided electromagnetic wave propagation has opened up the possibility of new quantum devices. These devices rely on quantum effects for their operation and are based on interferometric principles. Several switching devices have been proposed wherein one can control the relative phase difference between the two interfering paths by applying electrostatic potentials or magnetic fields. The transmission across these devices can be varied between zero and one (100 percent modulation), if the propagation takes place in the fundamental transverse mode (single channel regime). This requires that the Fermi energy should be between the ground and the first excited mode. Otherwise the mode mixing tends to average out the transmission oscillations. However, the proposed quantum devices are not very robust in the sense that the operational characteristics depend very sensitively on the material parameters. Incorporation of a single impurity, however weak, in the mesoscopic device may change non-trivially the interference of partial electron waves throughout the sample and hence electron transmission (operational characteristics across the sample). Such devices can be exploited only if we achieve the technology that can reduce or control the phase fluctuations to a small fraction of $2 \pi$.

We have studied the transmission across normal metallic loop connected to reservoirs by ideal wires in the presence of magnetic or $\mathrm{AB}$ flux. When electrons travel as evanescent modes in the loop we find that initial differential magnetoconductance is always negative and is unaffected by the presence of impurities in the loop [16]. Here transport arises in the presence of two non-classical effects namely, AB-effect and quantum tunneling. The above situation can arise in a system in which the transverse width of the loop is much less than the width of the ideal wires. Then due to the higher zero point energy arising from transverse confinement, fundamental subband minima in the loop will be at a higher energy than the value of the few subband minima in the ideal connecting wires. Then a situation can arise, where several propagating modes in the wire will have energy less than the minimum propagating subband energy in the loop. Thus the electron propagating in a fundamental subband of an ideal wire feels a barrier to its motion (arising solely due to the mismatch of the zero point energies) and thus the electron tunnels through across the loop (due to evanescent propagation) experiencing a higher effective potential barrier $V$. The fact of initial differential magnetoconductance being negative can be used for the operation of a quantum switch where the on and off states would correspond to transmission in the absence or presence of magnetic field respectively, i.e., the on state always has larger conductance than the off state. This difference in conductance can be made large by purposefully incorporating weak impurities in the arms of the connecting leads so long as they do not create resonant states in the system [17]. The robustness in the behavior of the initial differential magnetoconductance is related to the dominance of the first harmonic component in flux in the presence of evanescent modes only (as in the case of persistent currents discussed above). This is in contrast to the fact that the initial differential magnetoconductance for propagating modes can be either positive or negative and is very sensitive to small changes in geometric details and Fermi energy. 


\section{A new phase of the electron wave function}

In this section we discuss parity effects and observed quantum phase slips, a subject of ongoing current interest. States in a ring pierced by a magnetic flux exhibit strong parity effect [18]. There are two ways of defining this parity effect in the single channel ring (multichannel rings can be generalized using the same concepts). In the single particle picture (possible only in absence of electron-electron interaction) it can be defined as: states with an even number of nodes in the wave function carry diamagnetic currents (positive slope of the eigen energy versus flux) while states with an odd number of nodes in the wave function carry paramagnetic currents (negative slope of the eigen energy versus flux) [18]. In the many body picture (without any electron-electron interaction), it can be defined as: if $N$ be the number of electrons (spinless) in the ring, the persistent current carried by the $N$ body state is diamagnetic if $N$ is odd and paramagnetic if $N$ is even [18]. Leggett conjectured [19] that this parity effect remains unchanged in the presence of electron-electron interaction and impurity scattering of any form. His arguments can be simplified to say that when electrons move in the ring, they pick up three different kind of phases: 1) Aharanov-Bohm phase due to the flux through the ring, 2) statistical phase due to electrons being Fermions and 3) phase due to wave like motion of electrons depending on their wave vector. The parity effect is due to competition between these three phases along with the constraint that the many body wave function satisfy periodic boundary condition (which means if one electron is taken around the ring with the other electrons fixed, the many body wave function should pick up a phase of $2 \pi$ in all). Electron-electron interaction or simple potential scattering cannot introduce any additional phase although it can change the kinetic energy or the wave vector and hence modify the third phase. Simple variational calculations showed that the parity effect still holds [19]. Multichannel rings can be understood by treating impurities as perturbations to decoupled multiple channels, which means small impurities just open up small gaps at level crossings within the Brillouin zone and keep all qualitative features of the parity effect unchanged.

In a one dimensional (1D) system where we have a stub of length $v$ strongly coupled to a ring of length $u$ (the potential everywhere is uniform and all modes are propagating modes), we can have bunching of levels with the same sign of persistent currents [15]. This is essentially because if the Fermi energy is above the value where we have a node at the foot of the stub (that results in a transmission zero in transport across the stub), there is an additional phase of $\pi$ arising due to a slip in the Bloch phase [20,21] (Bloch phase is the third kind of phase discussed above, but the extra phase $\pi$ due to slips in Bloch phase is completely different from any of the three phases discussed above because this phase change of the wave function is not associated with a change in the group velocity or kinetic energy or the wave vector of the electron [20,21]). This is illustrated in Fig. 4 (a) and (b). The $y$-axis gives the phase of the electron wavefunction inside the ring, which is nothing but Bloch phase $\cos ^{-1} \operatorname{Re}[1 / t]$. The $\mathrm{x}$-axis gives the energy $E=k^{2}$. Fig 4 (b) exhibits discontinuous changes in the Bloch phase precisely as the energy crosses the transmission zeroes of the stub. In Fig 4 (a) the length of the stub and the ring is so tuned that the discontinuous phase slips cannot occur. Fig 4 (a) is similar to Fig 6 in Ref [18] where there is no violation of parity effect. But Fig 4 (b) exhibits violation of parity effect. A more detailed analysis can be found in Ref. [20]. It is worth mentioning that parameter values for which one can obtain no phase discontinuities as in Fig. 4 (a) forms a set of measure zero. A physical understanding of the phase slip can be obtained by mapping the 
stub to an effective potential of $V(k, x)=k \cot (k L) \delta(x)$. As the effective potential has discontinuities at $k L=\pi / 2,3 \pi / 2, \ldots$, at these energies the scattering phase and hence the Bloch phase of the electron in the ring will also exhibit discontinuities.

In an energy scale $\Delta_{u} \propto 1 / u$ (typical level spacing for the isolated ring of length $u$ ) if there are $n_{b} \sim \Delta_{u} / \Delta_{v}$ (where $\Delta_{v} \propto 1 / v$, the typical level spacing of the isolated stub of length $v$ ) such phase slips then each phase slip gives rise to an additional state with the same slope and there are $n_{b}$ states of the same slope or same parity bunching together with a phase slip of $\pi$ between each two of them [20]. Transmission zeroes is an inherent property of Fano resonance generically occurring in mesoscopic systems and this phase slip is believed to be observed [22-26] in a transport measurement [27]. The experiment [27] was done by embedding a quantum dot in one arm of an Aharonov-Bohm ring. The set up is an analog of the Young double slit experiment with a mica sheet on the path of an interfering beam, where one can estimate the phase acquired when light travels through the mica sheet by observing the change of interference pattern on the screen. In this case one can estimate the phase $\theta_{d}$ acquired by an electron in passing through the dot. The experiment was first interpreted in terms of Friedel sum rule in Ref. [28]. The dot contains many electrons that are strongly interacting with each other. As the Fermi energy sweeps through a resonance one additional electron is added to the dot and $\theta_{d}$ changes by $\pi$ as is expected from Friedel sum rule. The exact nature of this phase change could be explained from the theory of Bright-Wigner resonance. Now Friedel sum rule also suggests that no more $\pi$ phase shift should occur unless the next electron is added at the next resonance. But between two resonances an additional change of $\pi$ over a surprisingly small energy scale was observed in the experiment. This small energy scale is at least an order of magnitude smaller than any energy scale possible in the system or even the thermal broadening energy scale in the experiment. Initially it was thought [28] that may be there is some accidental charge addition into the system. For example it was argued [28] that the states of the ring may play a role if the states of the dot are coupled to the states of the ring. It was shown that charging of a ring state or addition of a charge in the ring can produce a sudden phase change also which can be misinterpreted as a phase change due to the dot. But repeated experiments showed that this sharp phase change is a very general feature occurring between every resonances and one can not take spurious charging effects to be a logical explanation. So it was felt that one has to find a new mechanism for this phase change that does not fit with the theoretical frame work of Friedel sum rule and Bright-Wigner resonance. We used the discontinuous phase change at the transmission zeroes of the stub structure to give such a new mechanism [22]. The stub was used as theoretical model and the exact geometry of the stub is not important [23]. The essential requirement is the degeneracy between a scattering state and a resonance state that yields a Fano resonance [23]. Since the quantum dot can support bound states and in a finite width wire these bound states are always degenerate with scattering states, we have to understand the phase changes in terms of Fano resonances [23]. Each of the Fano resonances have a zero-pole pair. Two consecutive resonances are largely separated by the charging energy of the dot. And hence there will be a phase change of $\pi$ at the pole according to Friedel sum rule because a charge is captured by the dot and a phase change of $\pi$ at the zero that will lie between two well separated poles. And hence this mechanism yields the basic feature of systematic phase changes as observed in the experiment. It also explains the extremely small energy scale over which the phase change between the poles occur. This explanation was further supported by Refs. [24-26]. A similar case was studied in Ref. [29] where 
they show the transmission zeroes and abrupt phase changes arise due to degeneracy of "dot states" with states of the "complementary part" and hence these are also Fano type resonances.

\section{Some consequences of parity violation}

In this section we will briefly discuss the results of Ref. [30]. Essentially it can be shown that break down of parity effect in the ring-stub system results in very non-trivial temperature dependence of persistent currents, a feature that may be exploited to experimentally study the effects of temperature on a quantum mechanical phenomenon like persistent currents.

We consider both the grand canonical case (When the particle exchange with a reservoir at temperature $T$ is present and the reservoir fixes the chemical potential $\mu$. In this case we will denote the persistent current as $I_{\mu}$ ) and the canonical case (When number $N$ of particles in the ring-stub system is conserved. In this case we will denote the persistent current as $I_{N}$ ). For the grand canonical case we suppose that the coupling to a reservoir is weak enough and the eigenvalues of electron wave number $k$ are not affected by the reservoir [18]. They are defined by the following equation [15]

$$
\cos (\alpha)=0.5 \sin (k u) \cot (k v)+\cos (k u),
$$

where $\alpha=2 \pi \phi / \phi_{0}$, with $\phi_{0}=h c / e$ being the flux quantum. Note, that Eq. (1) is obtained under the Griffith boundary conditions [9] which take into account both the continuity of an electron wave function and the conservation of current at the junction of the ring and the stub; and hard wall boundary condition at the dead end of the stub. Each of the roots $k_{n}$ of Eq.(1) determines the one-electron eigenstate with an energy $\epsilon_{n}=$ $\hbar^{2} k_{n}^{2} /(2 m)$ as a function of the magnetic flux $\phi$. Further we calculate the persistent current $I_{N / \mu}=-\partial F_{N / \mu} / \partial \phi$ [7], where $F_{N}$ is the free energy for the regime $N=$ const and $F_{\mu}$ is the thermodynamic potential for the regime $\mu=$ const. In the latter case for the system of noninteracting electrons the problem is greatly simplified as we can use the Fermi distribution function $f_{0}(\epsilon)=(1+\exp [(\epsilon-\mu) / T])^{-1}$ when we fill up the energy levels in the ring-stub system and we can write the persistent current as follows [18]

$$
I_{\mu}=\sum_{n} I_{n} f_{0}\left(\epsilon_{n}\right)
$$

where $I_{n}$ is a quantum mechanical current carried by the $n$th level and is given by [15]

$$
\frac{\hbar I_{n}}{e}=\frac{2 k_{n} \sin (\alpha)}{\frac{u}{2} \cos \left(k_{n} u\right) \cot \left(k_{n} v\right)-\left[\frac{v}{2} \operatorname{cosec}^{2}\left(k_{n} v\right)+u\right] \sin \left(k_{n} u\right)} .
$$

For the case of $N=$ const we must calculate the partition function $Z$ which determines the free energy $F_{N}=-T \ln (Z)$ 


$$
Z=\sum_{m} \exp \left(-\frac{E_{m}}{T}\right)
$$

where $E_{m}$ is the energy of a many electron level. For the system of $N$ spinless noninteracting electrons $E_{m}$ is a sum over $N$ different (pursuant to the Pauli principle) one-electron energies $E_{m}=\sum_{i=1}^{N} \epsilon_{n_{i}}$, where the index $m$ numbers the different series $\left\{\epsilon_{n_{1}}, \ldots, \epsilon_{n_{N}}\right\}_{m}$, where $n$ is level index and $i$ is the particle index. For instance, the ground state energy is $E_{0}=\sum_{n=1}^{N} \epsilon_{n n}$.

We show a non-monotonous temperature dependence of the persistent currents in this ballistic ring-stub system in the grand canonical $\left(I_{\mu}\right)$ as well as in the canonical case $\left(I_{N}\right)$. There is a crossover temperature $T^{*}$, below which persistent currents increase in magnitude with temperature while it decreases above this temperature. This is in contrast to persistent currents in rings being monotonously affected by temperature. $T^{*}$ is parameter dependent but of the order of $\Delta_{u} / \pi^{2} k_{B}$, where $\Delta_{u}$ is the level spacing of the isolated ring. For the grand canonical case $T^{*}$ is half of that for the canonical case. We also show that such a non-monotonous temperature dependence can naturally lead to a crossover from $\phi_{0} / 2$ periodicity to $\phi_{0}$ periodicity of the persistent currents as a function of temperature, where $\phi_{0}=$ hc/e. This is essentially because each of the harmonics can show an enhancement with temperature, at low temperatures, the crossover temperature for the $m$ th harmonic being approximately $T^{*} / \mathrm{m}$. Hence the second harmonic can peak at a lower temperature than the first and so can overtake the first harmonic in a certain temperature window. The temperature dependence of the fundamental periodicity is a very unique feature that cannot be seen in the ring system. The ring system can under certain circumstances show the $\phi_{0}$ periodicity as well as the $\phi_{0} / 2$ periodicity, but the periodicity remain unchanged with temperature. In the ring-stub system temperature enhancement of the first harmonic can be very robust and experimentally one can observe each of the harmonics separately.

To summarize, the ring-stub system has a lot of non-trivial temperature dependence of persistent currents which can provide some experimental motivation. The new phase discussed in the last section is the key source of the non-trivial temperature dependence.

\section{Dephasing of AB-oscillations due to spin-flip scattering in one arm of the AB-ring}

Understanding of dephasing and decoherence in mesoscopic systems connected with measurement and the quantum to classical transition is a subject of current interest. The importance of decoherence in realizing the classical world is well known [31]. The loss of interference (dephasing) in mesoscopic rings from the point of view of the trace left by the interfering particle on its environment or the effect of environment on the phase of the electron wavefunction has been clearly discussed in a seminal paper by Stern et. al. [32]. Our work [33] is in the close spirit to the above mentioned work of Stern et. al.. We have studied the transmission across a single channel AB-ring geometry using the quantum waveguide theory. In one of the arms (say upper arm) we have incorporated a spin half $(S=1 / 2)$ magnetic impurity. Electron interacts with this impurity via an exchange interaction of the type $J$ S.s, where $s$ is the spin of the electron and $J$ is the coupling strength. This interaction conserves the total spin $\mathcal{S}=S+s$. The magnetic impurity does not have its own dynamics. Depending on the initial direction of incident electron spin and 
that of the spin of the impurity spin-flip scattering takes place without exchange of any energy. Consider two partial waves going across once and initial spin is up, i.e., electron is in $s_{z}=+1 / 2$ state with magnetic impurity being in down $\left(S_{z}=-1 / 2\right)$ state. In this case the electron can get spin-flipped while traversing the upper arm, but no spin-flip occurs while traversing the lower arms. Thus, we would naively expect transmission amplitude for spin down case to vanish due to destructive interference. This is because looking at the spin of the magnetic impurity we would know which path the electron has taken. This is in close spirit to which-path detector or information models being studied in the quantum theory of measurement. However, this naive expectation turns out to be incorrect. This is because even after getting spin-flipped in the upper arm the electron may get reflected and finally traverse the lower arm and contribute to the spin down component of transmission. This study reveals several interesting features such as spin polarized transport (spin conductance or polarization) is asymmetric in magnetic field whereas two probe charge conductance is symmetric. There is no systematics in the harmonic components in flux of spin down and spin up components of transmission coefficient (owing to the sensitivity to the details of geometry and scattering strength). However, we have noticed that the amplitude of oscillations of the transmission coefficient (visibility) for the down spin is small as compared to the up spin. This clearly brings out the feature of dephasing in this simple model.

\section{Conclusion}

In conclusion, we have discussed above our own work on several of phenomenon arising basically due to quantum interference effects in the mesoscopic systems. For this purpose we have considered single channel case and various geometries. We have worked in the framework of free electron model. We have also suggested the means of probing the experimental manifestations of these phenomenon and their applications. A careful study of these phenomena in presence of inelastic scattering due to phonons and electron-electron interaction is called for.

\section{References}

* email: deo@boson.bose.res.in

+ email: jayan@iopb.res.in

[1] B. J. van Wees et al, Phys. Rev. Lett. 60, 848 (1988); D. A. Wharam et al, J. Phys. C 21, L209 (1988).

[2] R. A. Webb, S. Washburn, C. P. Umbach, and R. B. Laibowitz, Phys. Rev. Lett. 54, 2696 (1985); G. Timp, A. M. Chang, J. E. Cunningham, T. Y. Chang, P. Mankiewich, R. Behringer, and R. E. Howard, Phys. Rev. Lett. 58, 2814 (1987).

[3] L. P. Levy et al, Phys. Rev. Lett. 64, 2074 (1990); V. Chandrasekhar et al, Phys. Rev. Lett. 67, 3578 (1991).

[4] S. Washburn and R. A. Webb, Adv. Phy, 35 (1986) 375.

[5] M. Büttiker, Y. Imry, and R. Landauer, Phys. Lett. 96A, 365 (1983).

[6] D. Mailly et al, Phys. Rev. Lett. 70, 2020 (1993).

[7] N. Byers, C.N. Yang, Phys. Rev. Lett. 7, 46 (1961); F. Bloch, Phys. Rev. B 2, 109 (1970).

[8] M. Büttiker, Phys. Rev. B 32, 1846 (1985).

[9] S. Griffith, Trans. Faraday. Soc. 49, 650 (1953).

Pramana - J. Phys., Vol. 54, No. ?, February 2000 
[10] A. M. Jayannavar and P. Singha Deo, Phys. Rev. B 51, 10175 (1995).

[11] T. P. Pareek, P. Singha Deo and A. M. Jayannavar, Phys. Rev. B. 52, 14657 (1995).

[12] M. V. Moskalets, Euro. Phys. Lett. 41, 189 (1998).

[13] T. Choi, C. M. Ryu and A. M. Jayannavar, Int. Journ. of Mod. Phys. B 12, 2091 (1998); condmat/9808245.

[14] P. Singha Deo and A. M. Jayannavar, Mod. Phys. Lett. B 7, 1045 (1993).

[15] P. Singha Deo, Phys. Rev. B 51, 5441 (1995).

[16] A. M. Jayannavar and P. Singha Deo, Mod. Phys. Lett B, 8, 301 (1994).

[17] B. C. Gupta, P. Singha Deo and A. M. Jayannavar, Int. Journ. of Mod. Phys. B 10 3595(1996).

[18] H.F.Cheung, Y.Gefen, E.K.Riedel, W.H.Shih, Phys. Rev. B 37, 6050 (1988).

[19] A.J. Leggett in: Granular nano-electronics, eds. D. K. Ferry, J.R. Barker and C. Jacobony, NATO ASI Ser. B 251 (Plenum, New York, 1991) p. 297.

[20] P. Singha Deo, Phys. Rev. B 53, 15447 (1996).

[21] P. A. Sreeram and P. Singha Deo, Physica B 228, 345 (1996).

[22] P. Singha Deo and A. M. Jayannavar, Mod. Phys. Lett. B 10, 787 (1996).

[23] P. Singha Deo, Solid St. Communication 107, 69 (1998).

[24] C.M.Ryu et al, Phys. Rev. B 58, 3572 (1998); Hongki Xu et al, Phys. Rev. B, 57, 11903 (1998).

[25] H.-W.Lee, Phys. Rev. Lett., 82, 2358 (1999).

[26] T. Taniguchi and M. Büttiker, Phys. Rev. B 60, 13814 (1999).

[27] R. Schuster et al, Nature 385, 417 (1997).

[28] L. Yeyati and M. Büttiker Phys. Rev. B 52 R14360 (1995).

[29] J.Wu et al, Phys. Rev. Lett. 80, 1952 (1998).

[30] M. V. Moskalets and P. Singha Deo, cond-mat/9908411.

[31] W. H. Zurek, Phys. Today 44, 36 (1991).

[32] A. Stern, Y. Aharonov and Y. Imry, Phys. Rev. A 41 , 3436 (1990).

[33] D. Sahoo, S. K. Joshi and A. M. Jayannavar (to be published)

\section{Figure captions}

Fig. 1 (a) The thin lines represent single channel quantum wires supporting a ring. Through the center of the ring there is a magnetic flux $\phi$ perpendicular to the plane of the paper. The reservoir- 1 is at a chemical potential $\mu_{1}$ and the reservoir- 2 is at a chemical potential $\mu_{2}$. There is a delta function repulsive potential of strength $V$ at the site marked X.

Fig. 1 (b) The thin lines represent single channel quantum wires along with a ring. Through the center of the ring there is a magnetic flux $\phi$ perpendicular to the plane of the paper. The reservoir- 1 is at a chemical potential $\mu_{1}$ and the reservoir- 2 is at a chemical potential $\mu_{2}$. There is a delta function repulsive potential of strength $V$ at the site marked X. The two archs of the ring are of length $L_{1}$ (upper arch) and $L_{2}$ (lower arch). Current through $L_{1}$ is $I_{1}$ and that through $L_{2}$ is $I_{2}$.

Fig. 2 An ideal ring of length $L$ connected to an electron reservoir by an ideal wire. Along the thin lines the quantum mechanical potential is zero and along the thick lines it is $V$.

Fig. 3 A quantum ring connected freely to a finite quantum wire. Length of the the ring is $u$ and length of the wire is $v$.

Fig. 4 (a) Graphical solutions for the allowed modes in a ring-stub geometry for $v / u=0.2$

Fig. 4 (b) Graphical solutions for the allowed modes in a ring-stub geometry for $v / u=0.21$ 


$$
P_{H}
$$




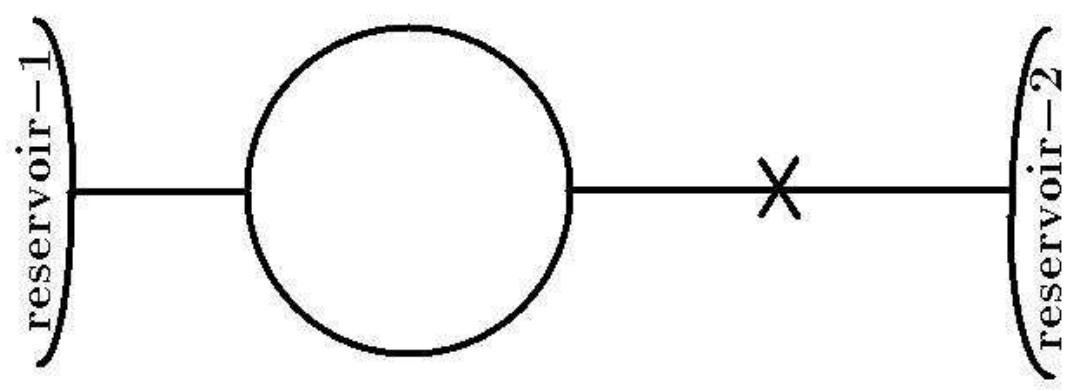




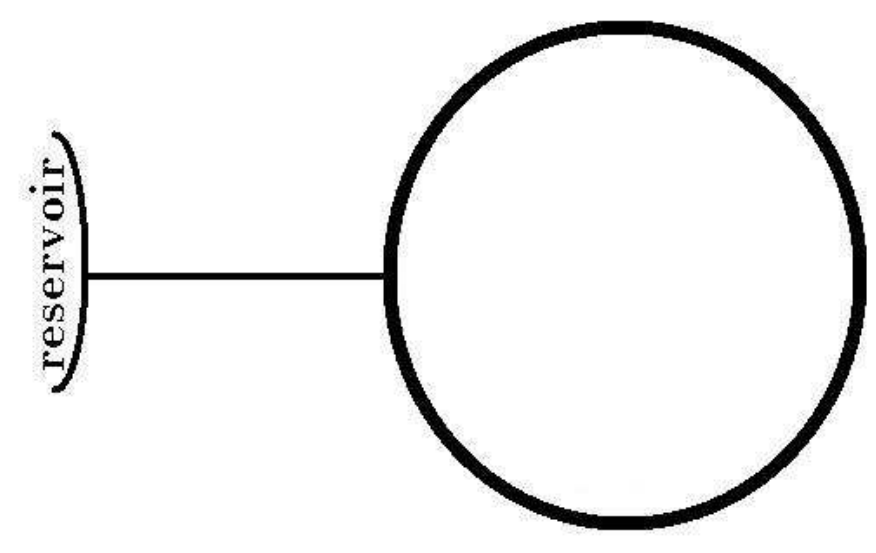




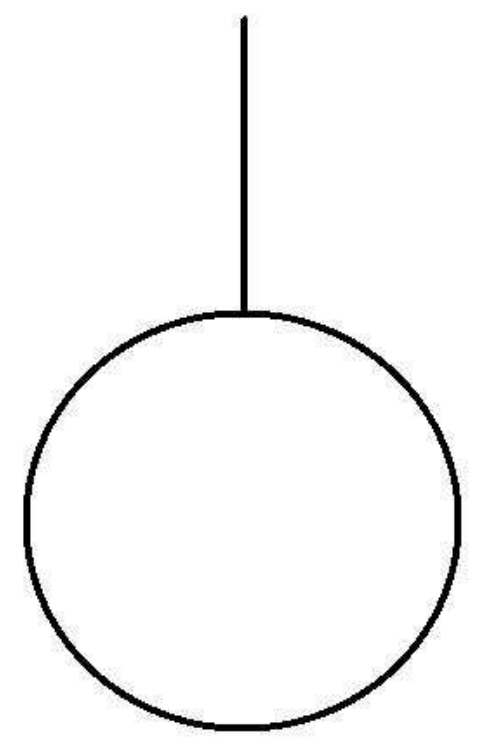

